\title{
Seasonal bud development in Maaka cherry
}

\author{
Anna Lokteva $^{* 1}$, Vladimir Simagin ${ }^{1}$ and Lyudmila Frolova ${ }^{2}$ \\ ${ }^{1}$ Central Siberian Botanical Garden SB RAS, 630090 Novosibirsk, Russia \\ ${ }^{2}$ Republican Scientific and Production Subsidiary Unitary Enterprise "Institute of Fruit Growing", \\ 223013, agro-town Samokhvalovichi, Belarus
}

\begin{abstract}
Wild relatives of fruit plants are an important reserve for introducing certain properties and traits into cultivated plants that significantly expand the range and facilitate the possibility of their use, since many wild species are sources of useful properties and traits that are poorly expressed or absent in cultivated species and varieties. One of these types is undoubtedly the Maaka.
\end{abstract}

\section{Introduction}

The development of horticulture in Russia is promising with the active involvement of new genetic sources, especially wild species, to create highly adapted local varieties. In the conditions of Western, Central and Eastern Siberia, the most winter-hardy type of cherry is the Maaka cherry. It is widely used in the landscaping of Siberian cities, forming longlived, large, profusely flowering trees with original shiny bark.

For the city of Novosibirsk, Maaka cherry is an introduced plant, originally imported from the Far East. It has adapted to our weather conditions, tolerates cold winters with a large snow cover and dry air, grows well and bears fruit in urban plantings. Basically, these are plants grown from seeds in nurseries, and planted in alleys and group plantings in city parks and squares. Some of the Maaka cherry seedlings have valuable decorative qualities.

\section{Material and methods}

Vegetative and generative buds of Maaka cherry were used as the object of the study. The material was collectedn from the collection at the experimental site of the Laboratory for the Introduction of Food Plants of the CSBS SB RAS. The buds for fixation were cut from the beginning of the spring (April) vegetation period until the end of September with an interval of 10 days.

Histological methods are used in many fields of science. They make it possible to study the internal structure of the organism, as well as to analyze the changes occurring in the plant organism at different stages of its development [1].

The preparation of permanent preparations was carried out using the method. N. P. Pausheva. The objects were fixed with the FAA retainer. The objects were poured into paraffin molds, which were cut on a microtome of Micg HM 325 with a thickness of 10-12

\footnotetext{
*Corresponding author: lokteva30@mail.ru
} 
microns. Complex staining with hematoxylin according to Ehrlich and alcyan blue was performed.

The preparations were analyzed using an Axioplan 2 imaging microscope (Carl Zeiss). In our studies, comparative morphological descriptions of the structure of the kidneys were carried out. In this article, the histological sections made from the beginning of the vegetation of plants and before leaving the pack in the studied samples are analyzed.

\section{Results and discussion}

For the first time, this species was attracted to hybridization with cherries by I. V. Michurin (1948). Later, his students and followers often used this species to create highly diseaseresistant varieties and rootstocks for cherries [2]. Recent studies have shown that the previously existing assignment of this species to the genus (subgenus) Cherry is erroneous, and it is advisable to transfer it to the genus (subgenus) Cherry $[3,4]$.

The natural range of the Maaka cherry is found in the Far East in the basins of the Ussuri and Amur rivers and in the eastern part of the Zeysko-Bureysky basin, as well as in the north-eastern regions of China, Japan, and the Korean Peninsula [5]. Thanks to modern research, the introduced range has greatly expanded within Russia.

On the territory of the city of Novosibirsk, the morphological variability of the flowers, fruits and leaves of the Maaka cherry was studied and its wide range of variability was shown for a number of characteristics, including those of value for practical use.

One of the rather conservative organs in flowering plants is the kidney. Therefore, a comprehensive study of the features of the morphological structure of flower buds in representatives of plum trees is important in the development of a natural classification of species and varieties of this group [6].

During ontogenesis, different types of buds are formed on the fruit tree: apical and lateral, which can be growth, flower and flower-growth. In stone crops, some buds on the shoot are flowering, others are vegetative. Inside each flower bud are the rudiments of only the flowers, and in the vegetative bud are the rudiments of the leaves. Such kidneys are specialized, they are often called simple. From some buds, only flowers develop (from the lateral ones), from others (apical ones), a shoot develops. The Maaka tree belongs to woody plants with a long growing season. In the conditions of the city of Novosibirsk, the spring awakening of the buds occurs in mid-April, flowering occurs on May 20-25, lasts 10-12 days. The fruit ripens after July 20 (July 20-25) and lasts until the end of September. The beginning of autumn leaf color occurs in mid-September, leaf fall occurs after October 10.

Our research and analysis of the literature indicate the initial external uniformity of the morphological structure of all kidneys. On the longitudinal sections of each bud, the growth cone with the adjacent leaf rudiments and covering scales is clearly discernible. In the axils of each leaf bud, there is an axillary cone of growth. The flower buds of the Maaka cherry are formed separately from the leaf buds.

The laying of vegetative and generative buds in Maka cherries begins in late May and early June, and closer to autumn, secondary, lateral buds are formed in the axils of simple leaves, often two at a time. In generative buds, the formation of the inflorescence occurs after July 20.

On the longitudinal sections in the center of each bud, the cone of growth is clearly visible under the microscope, with the adjacent rudiments of leaves and covering scales. In the sinus of each rudimentary leaf there is an axillary cone build-up. On the histological sections, the specifics of the external design of the flower bud are clearly visible. In most fruit plants, including cherry trees, inflorescences consisting of several flowers are formed in the flower buds (Fig). 


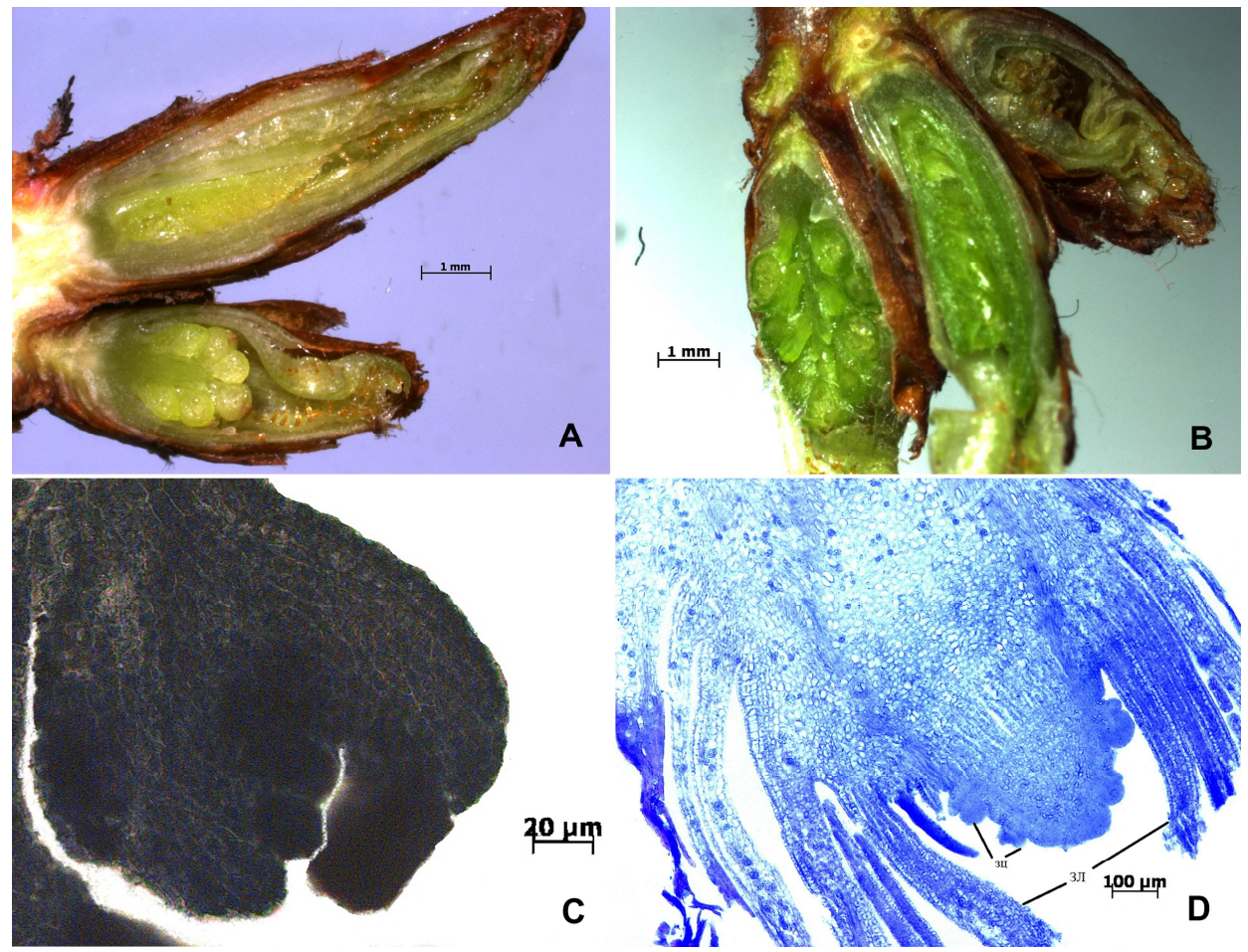

Fig. A kidney slice in April, B section of the bud May, C. Rudimentary flower, D. Flower bud with rudimentary leaves (zl) covering the inflorescence, a section of the inflorescence with rudimentary flowers (zc) located on it.

\section{Conclusion}

Generative and vegetative buds have a similar structure in all the samples considered by us. In the flower buds of cherries, a brush of several flowers is formed.

The life cycle of each kidney includes the growth of its organs, which can be vegetative and generative. The ontogenesis of a fruit plant consists of morphophysiological cycles, in which specific features of growth, the formation of buds and flowers are manifested. The life cycle of the axillary cone from its appearance in the flower-growth buds of cherries, to the formation of the rudiments of flowers and their flowering usually lasts two years. In general, the same anatomical structure of the stem was observed on the histological sections: dense integumentary layers, the conducting system, and the core.

\section{Acknowledgements}

This work was supported by the state project number: AAAA-A21-121011290027-6 for the Central Siberian Botanical Garden (CSBG) SB RAS). Materials of the bioresource scientific collection of the CSBG SB RAS "Collections of living plants in open and closed ground", USU No. 440534 were used.

\section{References}

1. N. I. Belozor, Works on applied botany and selection, 77 (1983) 
2. V. L. Vitkovsky, K. Yu. Mostolovitsa, Z. M. Gavrilina, Collection of scientific papers on applied botany, genetics and breeding, 106 (1986)

3. V. S. Simagin, G. V. Eremin, Flora and vegetation of Altai, 4(1) (1999)

4. G. V. Eremin, V. G. Eremin, Scientific works of SKFNTsSVV, 25 (2019)

5. Z. P. Pausheva, Workshop on plant cytology (Moscow, Kolos, 1988)

6. R. H. Wetmore, C. W. Wardlaw, Annu. Rev. Plant Phisiol., 2 (1951) 